\title{
In situ Molecular Imaging of Heterogeneous Catalytic Processes in Liquid Environments
}

\author{
Pratibha L. Gai \\ DuPont Central Research and Development, Wilmington, DE 19880-0356, U.S.A. and \\ Department of Materials Science, University of Delaware, U.S.A.
}

Understanding reacting materials at the atomic level is important for the design and development of new generation catalysts, new routes for polymers and green technological processes. Many commercial polymerization reactions and nanomaterials are derived from solutions and wet chemical methods. Probing reactions in solutions at the molecular level is therefore of great importance in the development of advanced catalytic process technologies. To probe gas molecule-solid catalyst reactions directly at the atomic level, we have pioneered the development of in-situ atomic resolution environmental transmission electron microscopy method (referred to as atomic resolution-ETEM, or as environmental-HRTEM) [1,2]. We have recently advanced the in situ technique to probe dynamic catalysis in liquid environments, and this method is referred to as wet-ETEM [3]. Our wet-ETEM capability allows high resolution imaging in liquids, with capabilities for heating samples to reaction temperatures. Here we demonstrate molecular imging in liquid environments. We illustrate this development with examples of the catalytic hydrogenation of nitriles in the liquid phase and in the manufacture of nanomaterials.

Using wet-ETEM, we have investigated the hydrogenation of adiponitrile $\left(\mathrm{NC}\left(\mathrm{CH}_{2}\right)_{4} \mathrm{CN}\right)$ in liquid phase over cobalt modified ruthenium nanocatalysts supported on rutile titania. For the studies, the catalysts were immersed in $\mathrm{ADN}$ liquid (in methanol and $0.15 \mathrm{wt} \% \mathrm{NaOH}$ solvent) in a liquid injection sample stage with heating capabilities, which we have developed [3]. Upto microliters of the liquid were injected over the catalysts. Flowing hydrogen was passed over the samples simultaneously and the sample was heated to $\sim 100{ }^{\circ} \mathrm{C}$. Dynamic reactions were recorded in situ under the operating conditions. During the studies, we have observed that, bis-hexamethylene triamene (BHMT) is formed, along with the main product of hexamethylene diamene $\left(\mathrm{H}_{2} \mathrm{~N}\left(\mathrm{CH}_{2}\right)_{6} \mathrm{NH}_{2}\right)$ or HMD. Molecular imaging and electron diffraction under liquid environments have revealed the structure of BHMT. Figure 1 shows the nanocatalyst at room temperature (RT). Fig 2(a) and (b) show the catalyst in ADN liquid at RT and the formation of BHMT at the catalyst surface at $100{ }^{\circ} \mathrm{C}$, respectively. Figure 2(c) shows the enlarged area from Figure 2(b), revealing the lattice layers in BHMT organic molecules. The dynamic images show that it is crystalline with the lattice spacing of about 5.6 angstroms. Figure 3 shows the formation of HMD at $100{ }^{\circ} \mathrm{C}$. Wet-ETEM is also used to study the growth of gold nanorods [Figure 4]. The wet-ETEM method is opening up striking new opportunities in the direct molecular studies of dynamic polymerization reactions and in biomolecular nanotechnological applications.

References:

1. P.L. Gai et al., Science. 267 (1995) 666.

2. E,D. Boyes and P.L. Gai, Ultramicroscopy. 67 (1997) 219.

3. P.L. Gai, Microscopy and Microanalysis. 8 (2002).

4. L.G. Hanna, F.G. Gooding, K. Kourtakis and S. Ziemecki are thanked. 


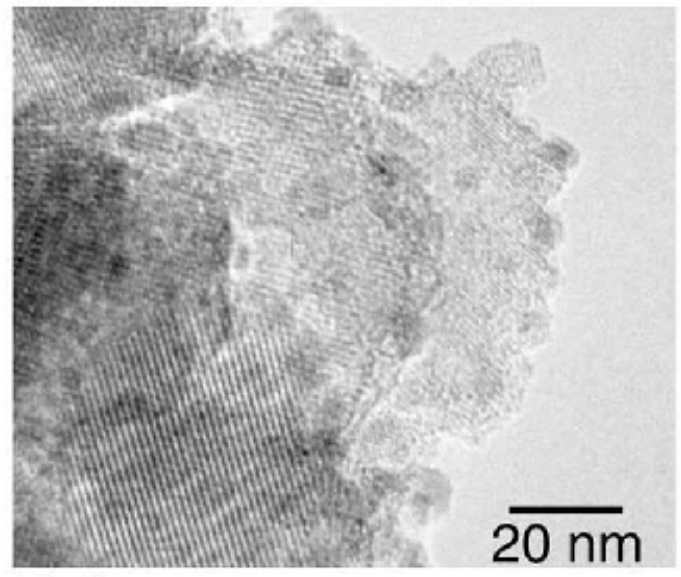

Fig 1

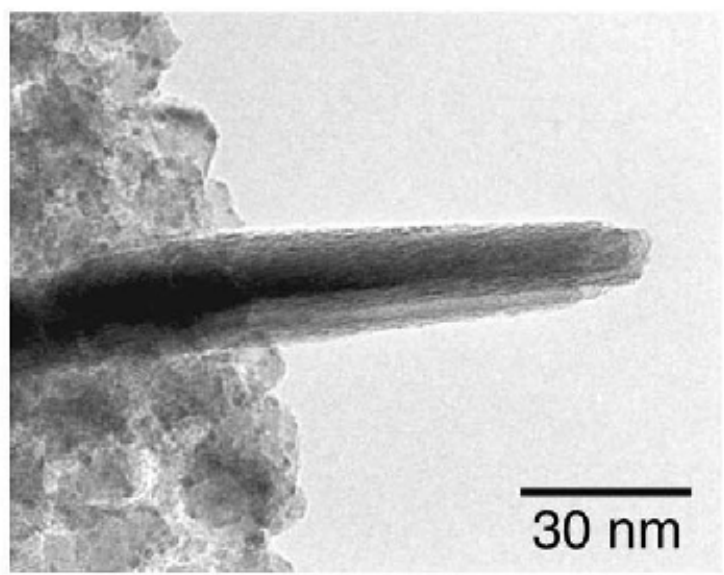

Fig $2 b$

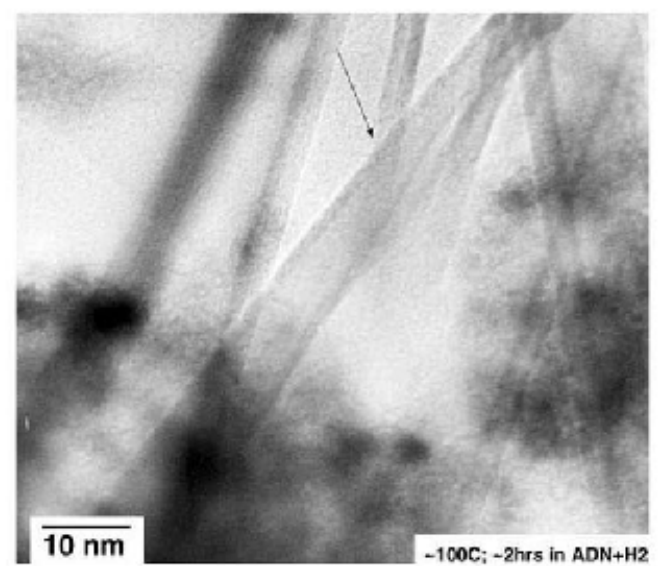

Fig 3

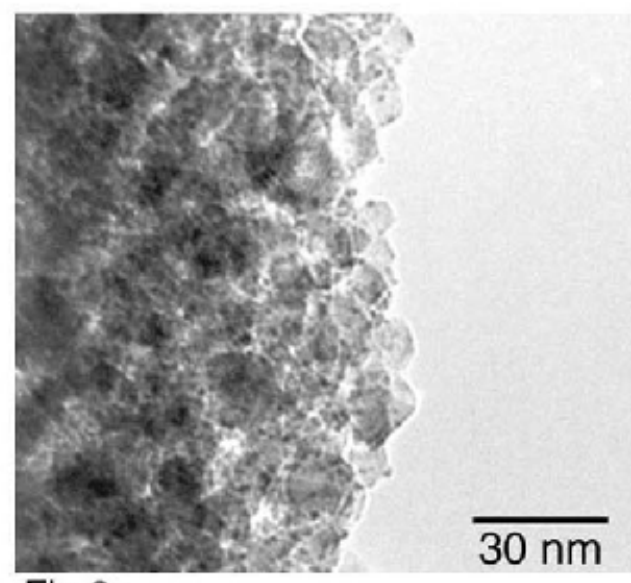

Fig $2 a$

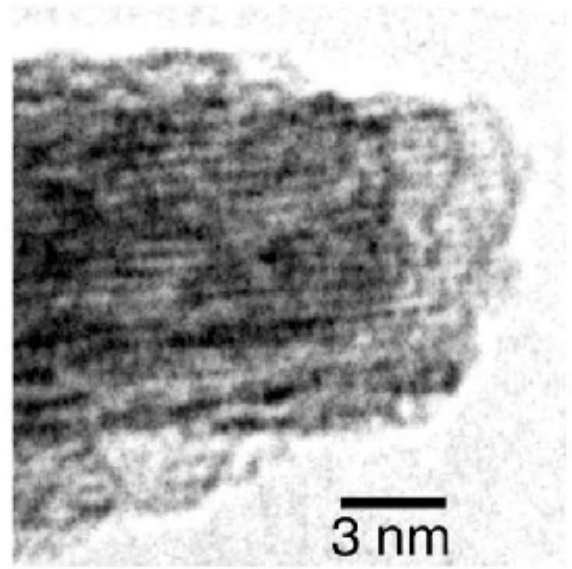

Fig 2c

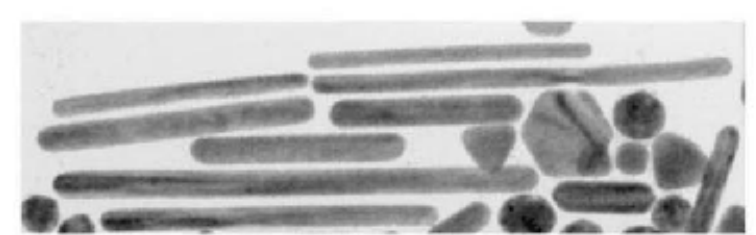

Fig 4

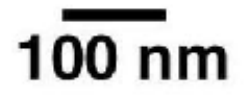

Figure captions:

FIG.1. Heterogeneous cobalt modified Ru/titania nanocatalyst.

FIG.2(a). Wet-ETEM of the nanocatalyst in ADN liquid and $\mathrm{H}_{2}$ gas at RT;

FIG.2(b). Wet-ETEM of Fig 2(a) at $\sim 100^{\circ} \mathrm{C}$, showing BHMT molecular structure;

FIG.2(c). Enlargement of FIG. 2(b) showing the molecular structure.

FIG.3. HMD formation; FIG 4: high aspect ratio nanorods. 